systematischer Folge wechseln, bieten diesen Studirenden Gelegenheit, die bauptsächlichsten Beobachtungen, auf welche in der Vorlesung Bezug genommen wird, selbst zu machen. Der allgemeine Gang ist dabei der, dass mit der Messung von Überführungszahlen und Leitfähigkeiten begonnen wird. Danach wird die Bestimmung von elektromotorischen Kräften und Zersetzungsspannungen vorgenommen, und es werden Normalelemente und Accumulatoren, letztere unter Benutzung käuflicher ungepasteter Gitterplatten hergestellt und untersucht. Daran schliessen sich galvanostegische und elektroanalytische Versuche, die ziemlich kurz behandelt werden, da hier einerseits der Besuch industrieller Etablissements, andererseits der analytische Unterrichtsgang des wissenschaftlich-chemischen Laboratoriums ergänzend zur Seite stehen. Im weiteren Fortgang werden die Darstellung von Natrium, Aluminium, Magnesium, die Entzinnung von Weissblech und die Raffination von Kupfer, sowie ähnliche elektrometallurgische Processe demonstrirt. Die Erzeugung des Carbides mit Widerstands- und Lichtbogenöfen in den üblichen Laboratoriumsdimensionen wird vorgeführt und die Verwendung des Acetylens als Lichtgeber durch Bestimmung seines Leucht- und Carburationswerthes erläutert. Weiter wird die Erzeugung von Bleichlaugen und ron Chlorat, die Kochsalzelektrolyse unter Sonderung von Anoden- und Kathodenproducten messend verfolgt. Als letztes Thema wird die Darstellung wichtiger anorganischer und organischer elektrochemischer Processe und die Ozongewinnung behandelt.

Der Versuch, diese Unterrichtsformen durch ein Colloquium zu ergänzen, in welchem während des Winters van t'Hoff's chemische Dynamik, im Sommer die neuere PatentLitteratur und Mittheilungen technischer Fachzeitschriften erörtert werden, hat sich in den letzten Semestern mit gutem Erfolg durchführen lassen.

\section{Verfahren, Metakresol in Kresolgemischen zu bestimmen.}

Von Dr. F. Raschig-Ludwigshafen a. Rh.

Schon seit einer Reihe von Jahren wird als Ersatz der Pikrinsäure, die unter dem Namen „Melinit“, "Lyddit" etc. zum Füllen von Sprenggeschossen dient, ein "Cresylit" genanntes Product angewandt, welches beim Behandeln des Kresols, wie es in der Carbolsäurefabrikation abfällt und billig verkauft wird, mit Salpetersäure entsteht. Dieses
Kresol besteht aus einem Gemisch der drei bekannten Isomeren, $0^{-}, \mathrm{m}^{-}$und $\mathrm{p}$-Kresol; das daraus entstebende Nitroproduct ist aber ausschliesslich Trinitro-m-Kresol; 0 - und p-Kresol verbrennen bei der Nitrirungsart, die hier in Betracht kommt, nämlich in der Siedehitze und bei Gegenwart von Salpetersäureüberschuss, vollständig zu Oxalsäure.

Das Kresol des Handels wird im Mittel 40 Proc. o-Kresol, 35 Proc. $\mathrm{m}-\mathrm{K}$ resol und 25 Proc. $p$-Kresol enthalten und liefert rund 60 Proc. Trinitro - $m-$ Kresol. Dabei gehen grosse Mengen von Salpetersäure verloren, weil sie zur Oxydation des $o^{-}$und $\mathrm{p}$-Kresols aufgebraucht werden. Man kann durch eine sehr sorgfältige und oft wiederholte fractionirte Destillation aus dem Kresolgemisch das bei $188^{\circ}$ siedende o-Kresol vollständig abscheiden und erhält dann ein Gemisch von rund 60 Proc. $m-K r e s o l$ und 40 Proe. p-Kresol. Diese beiden sieden bei fast genau der gleichen Temperatur, bei $200^{\circ}$ und $199,5^{\circ}$; sie lassen sich also durch Fractioniren nicht mehr trennen. Aber schon dieses Gemisch der zwei Kresole zeigt gegenüber dem oben genannten aus dreien so grosse Vortheile es liefert etwa 100 Proc. Trinitro-m-Kresol und gebraucht dabei weniger Salpetersäure dass es von den betreffenden Fabriken gern vorgezogen und höher bezablt wird.

Um beim Handel mit solchen Fabrikaten, deren Werth sich nach ibrem Metakresolgehalt bestimmt, Differenzen nach Möglichkeit zu vermeiden, habe ich ein Verfahren ausgearbeitet, das Metakresol in derartigen Gemischen zu bestimmen. Dieses Verfahren ist in meiner Fabrik seit $1^{1 / 2}$ Jahren im regelmässigen Gebrauch; es sind schon Tausende von Metakresolbestimmungen danach ausgeführt worden, und es hat sich in der ganzen Zeit als zuverlässig und hinreichend genau bewährt. Es ist ferner leicht und schnell auszuführen, erfordert wenig Apparatur und macht nur geringe Kosten.

Die Methode beruht auf der erwähnten Eigenschaft des Kresolgemisches, beim Behandeln mit einem Überschuss von Salpetersäure in der Siedebitze ausschliesslich Trinitro$\mathrm{m}$-Kresol zu liefern, welches sich, als fast ganz unlöslich in Wasser, leicht abscheiden, trocknen und wiegen lässt. Es waren demnach die Versuchsbedingungen so anzuordnen, dass unter allen Umständen, bei grossem und bei geringem Gehalt an $m$-Kresol und gleichgiltig, ob die Beimischung vorwiegend aus 0 -Kresol oder aus $\mathrm{p}$-Kresol bestand oder beide in grossen Mengen enthielt, die Mengen des abgeschiedenen Trinitro-m-Kresols genau proportional dem m-Kresolgebalt ausfielen. Dazu gehört erstens, dass man das 
Kresol vor dem Nitriren sulfurirt und dafür sorgt, dass der. Sulfosäure auch nicht mehr die geringste Menge freien Kresols beigemischt ist und zweitens, dass die Gesammtmenge der Sulfosäure möglichst schnell und bei Gegenwart eines Überschusses von Salpetersäure auf die Siedetemperatur des $\mathrm{Ge}-$ misches, die zwischen 110 und $115^{\circ}$ liegt, kommt. Um der ersten Forderung zu genügen, reicht es nicht aus, wie vielfach geglaubt wird, das Kresol mit concentrirter Schwefelsäure einfach zu mischen; denn trotzdem dabei die Temperatur auf $50-60^{\circ}$ steigt, bleibt auch bei Schwefelsäureüberschuss leicht ein Theil des Kresols unsulfurirt. Man muss vielmehr mindestens eine Stunde lang Kresol mit Schwefelsäure auf 95-100 ${ }^{\circ}$ erhitzen. Zur Erfüllung der zweiten Bedingung mischt man die Sulfosäure, welche zu diesem Behufe vorher abzukühlen ist, mit einer Menge Salpetersãure, die mehr als ausreicht, um alles. Kresol zu nitriren bez. zu oxydiren, die aber andererseits so klein ist, dass die bei der Reaction entstehende Wärme im Stande ist, das ganze Gemisch in Zeit von 1 bis $1^{1 / 2}$ Minuten auf seine Siedetemperatur aufzuheizen. Diese Menge beträgt für $10 \mathrm{~g}$ Kresol $90 \mathrm{ccm}$ einer Salpetersäure von $40^{\circ}$ Bé.

Ich verfahre folgendermaassen:

Genau $10 \mathrm{~g}$ Kresol werden in einen kleinen Erlenmeyer-Kolben gewogen und mit $15 \mathrm{ccm}$ gewöhnlicher Schwefelsäure von $66^{\circ}$ Bé. gemischt. Der Kolben bleibt dann mindestens eine Stunde in einem durch Dampf geheizten Trockenschrank stehen. Alsdann giesst man seinen Inhalt in einen weithalsigen Kolben von etwa $1 l$ Fassungsraum und kühlt diesen unter Umschwenken an der Wasserleitung ab. Dabei legt sich die in der Wärme dünnflüssige Sulfosäure als dicker Syrup an die Wände des Literkolbens.

Nunmehr giesst man in den ErlenmeyerKolben, welcher zur Sulfurirung diente und dem noch geringe Reste der Sulfosäure anhaften, zum Ausspülen dieser Reste $90 \mathrm{ccm}$ gewöhnliche Salpetersäure $40^{\circ}$ Bé., bringt durch Umschwenken die Sulfosäurerückstände in Lösung und giebt sodann dieses ganze Quantum Salpetersäure auf einmal in den Literkolben. Dieser wird dann s of ort kräftig umgeschüttelt, bis alle Sulfosäure gelöst ist, was etwa 20 Secunden dauern mag. Dann stellt man den Kolben sogleich unter einen Abzug. Nach Verlauf von ungefäbr 1 Minute tritt eine heftige Reaction ein; der Inhalt kommt in lebhaftes Kochen, wobei viel rothe Dämpfe entweichen; dann trübt sich die bis dahin klare Flüssigkeit plötzlich, Öltropfen von Trinitrokresol scheiden sich aus und sammeln sich am Boden, und nach 5 Minuten scheint die ganze Reaction beendet. Man lässt aber noch mindestens fernere 5 Minuten stehen, weil doch noch geringe Nachnitrirung stattfindet; alsdann giesst man den ganzen Kolbeninbalt in eine Schale, die bereits $40 \mathrm{ccm}$ Wasser enthält und spült mit weiteren $40 \mathrm{ccm}$ Wasser nach. Bei diesem Mischen mit Wasser erstarrt das Öl unter Aufquellen und Entweichen nitroser Gase zu einem Krystallbrei von Trinitro-m-Kresol. Dieser bleibt bis zum völligen Erkalten der Flüssigkeit, mindestens zwei Stunden, stehen, wird dann mit einem Pistill grob zerdrückt und auf ein papiernes Saugfilter gebracht. Das Filtriren verläuft sehr schnell; man wäscht mit $100 \mathrm{ccm}$ Wasser, welche man am besten aus einem in eine Spitze ausgezogenen Trichter auf die Krystalle fliessen lässt, nach, trocknet mit dem Filter bei $95-100^{\circ}$ und wiegt mit $\mathrm{ihm}$, wobei man ein Filter von gleicher Grösse als Gegengewicht benutzt.

Bei einiger Übung ist man in 5 Stunden mit der Bestimmung fertig und kann leicht 10 bis 20 dieser Analysen neben einander machen.

Chemisch reines Metakresol liefert, auf diese Weise behandelt, genau 17,4 g Trinitrokresol und es ist durch eine grosse Anzahl von Versuchen nachgewiesen worden, dass die verschiedenartigsten Gemische von mKresol mit p-Kresol, mit o-Kresol und mit beiden stets auf 1 Proc. m-Kresol 1,74 Proc. Trinitrokresol geben. Ja selbst wenn man $9 \mathrm{~g}$ Metakresol mit $1 \mathrm{~g}$ Phenol gemischt so behandelt, erhält man genau $9 \times 1,74=15,6$ Nitroproduct. Wahrscheinlich bleibt die aus dem Phenol unter diesen Umständen entstehende Pikrinsäure hier in Lösung; bei mehr als 10 Proc. Phenolgehalt aber scheidet sie sich mit dem Trinitrokresol aus und erböht dessen Gewicht; auf stark phenolhaltige Kresolgemische ist das Verfabren demnach nicht anwendbar. Solche kommen aber in der Regel im Handel nicht vor und in ihnen verräth sich das Phenol auch sebr leicht durch seinen Siedepunkt, ferner auch dadurch, dass das Nitroproduct im Trockenschrank bei $95-100^{\circ}$ nicht fest bleibt, sondern zerfliesst oder wenigstens zu einem weichen Brei wird.

Auch Xylenole, die schon häufiger im Kresol des Handels zu finden sind, verrathen sich in derselben Weise; das Nitroproduct zerfliesst in der Wärme oder es will sogar in der Kälte nicht erstarren. Ein Kresol dagegen, welches in seiner allergrössten Menge zwischen 190 und $200^{\circ}$ destillirt und 
demnach kaum Phenol noch Xylenole enthält, giebt bei Innehaltung obiger Vorschriften stets einen hellgelben Krystallkuchen, dessen Gewicht, durch 1,74 dividirt, direct den Metakresolgebalt angiebt. Die Resultate schwanken nicht über 1 Proc. nach oben und nach unten.

Auf einen Umstand muss ich noch hinweisen. Die gewäblte Salpetersäuremenge ist erheblich grösser als nöthig wäre, um Alles zu nitriren und zu oxydiren, und man hat bei den Durchschnittskresolen von 35 Proc. bis zu 60 Proc. Metakresol auch schon mit $70 \mathrm{ccm}$ gute Resultate. Aber in diesem Falle tritt die Reaction oft so schnell ein, dass man kaum Zeit hat, Sulfosäure und Salpetersäure gründlich zu mischen und den Kolben fortzustellen; ja es ist einige Male vorgekommen, dass das Sieden so plötzlich und stürmisch anfing, dass der Kolben auseinander flog. Ich empfehle daher, von den vorgeschriebenen $90 \mathrm{ccm}$ Salpetersäure nicht abzugehen und darauf zu achten, dass der Kolben wenigstens $1 l$ Inhalt und einen weiten Hals habe. Auch ist es nöthig, dass die ganze Salpetersäuremenge auf einmal und schnell zur Sulfosãure gegeben werde; lässt man langsam zufliessen, so fängt die Reaction leicht schon an, bevor alle Säure im Kolben ist.

\section{Zur Untersuchung von Briquettepech.}

$$
\text { Von Dr. J. Klimont. }
$$

(Aus dem Laboratorium der K. K. priv. DonauDampfschiffahrtsgesellschaft.)

Für die Beurtheilung eines Steinkohlentheerpeches behufs Verwendbarkeit bei der Briquettefabrikation spielt nebst der Bindekraft die Erweichungstemperatur die wichtigste Rolle. Zur Bestimmung dieser Temperatur wurden bereits viele Vorschläge gemacht; allein den sichersten Anhaltspunkt gewährt noch immer die Methode von $\mathbf{E}$. Schenk zu Schweinsburg ${ }^{1}$, nach welcher eine schnabelförmig gebogene Röhre mit fein gestossenem Peche gefüllt und auf letzteres ein Tropfen Quecksilber gebracht wird. Die Temperatur, bei welcher das Aufschwellen des Peches stattfindet, nennt Schenk den "Schmelzpunkt", und diejenige Temperatur, bei welcher der Quecksilbertropfen untersinkt den „Flüssigkeitspunkt“.

Ohne näher darauf eingehen zu wollen, ob für die Beurtheilung eines Peches die Auseinanderbaltung dieser Temperaturunter-

1) Österr. Zeitschrift f. Berg- u. Hüttenwesen. 1890,463 schiede zweckmässig und nothwendig ist, muss hervorgehoben werden, dass die $\mathrm{Me}$ thode von Schenk nur in der Hand solcher Analytiker, welche dieselbe häufig handhaben, zuverlässige Resultate ergiebt. Es ist nämlich sowohl die Anschwellung als auch das Untersinken des Quecksilbertropfens deshalb schwer erkennbar, weil der feine Pechstaub die Oberfläche des Quecksilbers überzieht, so dass für das ungeübte Auge keinerlei Vorgänge wahrnehmbar sind.

Da aber andererseits für die Briquettefabrikation die zuverlässige Feststellung der Constanten eines Peches ungemein wichtig ist, so war ich darauf bedacht, eine Methode auszuarbeiten, die es gestattet, leichter als durch die bisherigen Verfahren und sicher die Temperaturen, bei welchen Erweichung und Verfüssigung erfolgt, zu erkennen.

Zunächst sei bemerkt, dass bei einem Peche, welches ein Gemisch der verschiedensten chemischen Individuen vorstellt, von einem Schmelzpunkt im engeren Sinne des Wortes wohl nicht die Rede sein kann. Vielmehr müsste zur Charakterisirung der Verflüssigung der ganze $10-12^{0}$ umfassende Vorgang des Übergehens vom festen in den flüssigen Aggregatzustand notirt werden. Allein die Praxis erfordert schärfere Zahlen; sie will den Moment des Anfangs und das Ende der Verflüssigung festgebalten wissen, und darum habe ich im Princip mich den Anschauungen Schenk's angeschlossen, welcher einen Schmelz- und einen Flüssigkeitspunkt normirt.

Diese Daten lassen sich auf zweierlei Weise durch die folgenden einfachen Versuche fixiren:

1. Man beschickt ein gewöhnliches Reagensrohr mit feingepulvertem Pech derart, dass man letzteres durch einen Trichter mit langem Rohre einfüllt. Sodann stösst man das Rohr mit dem Boden sanft so lange auf den Tisch, bis das Pulver zu einer compacten Masse zusammengeklopft ist, welche lediglich die Rundung des Reagensrohres ausfüllt. Nun wird das Reagensrohr, falls man es mit Weich- oder mittelhartem Pech zu thun hat, an einem Drahte neben ein Thermometer derart in ein Becherglas mit Wasser gehängt, dass die Thermometerkugel sich mit dem Pech in gleicher Höhe befindet. Bei Hartpech wird die Bestimmung zweckmässig im Glycerinbade vorgenommen. Mit der $2 \mathrm{~cm}$ hohen Flamme eines Bunsenbrenners erwärmt man nun langsam. Sobald am Rande die Pechstäubchen zu glänzenden kleinen Kügelchen zusammenschmelzen und der bisher matte Pechstaub an der Glaswand glänzend wird, beginnt der Schmelzpro- 